\title{
CASE REPORT \\ Body composition changes after 12 months of FES cycling: case report of a 60-year-old female with paraplegia
}

\author{
DR Dolbow ${ }^{1,2}$, AS Gorgey ${ }^{1,2}$, DR Gater ${ }^{1,2}$ and JR Moore ${ }^{1}$
}

Study design: Single-subject (female, 60 years of age) case.

Objectives: The purpose of this case report is to document body composition changes in a 60-year-old female with chronic paraplegia after 12 months of home-based functional electrical stimulation lower extremities cycling (FES-LEC).

Setting: Home-based FES-LEC with internet connection. Southeastern United States.

Methods: FES-LEC three sessions per week for 12 months in participant's home and monitored by the research staff via internet connection. Pre- and post-exercise program testing for body composition including percent body fat, fat mass (FM), lean mass (LM) and whole-body bone mineral density (BMD) via dual-energy $x$-ray absorptiometry (DXA).

Results: There was a $7.7 \%$ increase in total body LM and a $4.1 \%$ increase in legs $L M$. There was a $1.2 \%$ decrease in total body FM and a $9.9 \%$ decrease in legs FM. Percent body fat decreased from 48.4 to 46.3 and whole-body BMD was increased from 0.934 to 1.023, which resulted in an improvement in the DXA T-score from -2.4 to -1.3 .

Conclusion: Positive body compositional changes during this study support the idea that long-term FES-LEC can help restore healthier ratios of LM and FM and possibly decrease the risk of associated diseases. Increased whole-body BMD provides hope that long-term FES-LEC may be beneficial regarding bone health.

Spinal Cord (2014) 52, S3-S4; doi:10.1038/sc.2014.40

\section{INTRODUCTION}

Spinal cord injury (SCI) causing paraplegia or tetraplegia routinely results in dramatic changes in body composition, that is, decreased muscle mass, decreased bone mass and increased fat mass (FM). ${ }^{1,2}$ These body composition changes have been linked to increased risk of cardiovascular diseases, diabetes mellitus, metabolic syndrome and osteoporosis. ${ }^{1,2}$ Compounding the problem further, muscle paralysis limits the number of physical activity options resulting in extreme sedentary lifestyles. However, with new technological innovations such as internet-monitored functional electrical stimulation lower extremities cycling (FES-LEC), persons with SCI not only have the option to increase physical activity levels but also to exercise paralyzed muscles during the activity. FES-LEC has been reported as one activity that may help decrease the negative body composition changes after SCI; however, the long-term effects have not been fully examined. ${ }^{3}$ The authors could find no studies reporting home-based FES-LEC monitored via the internet over a 1 year duration.

The purpose of this case report is to document body composition changes in a 60-year-old female with chronic paraplegia after 12 months of home-based FES-LEC.

\section{STUDY DESIGN/METHODS}

A 60-year-old female 2 years post T6 American Spinal Injury Association Impairment Scale A SCI participated in home-based FES-LEC averaging 2.9 sessions per week over 12 months. The participant utilized an RT300 FES cycle (Restorative Therapies, Baltimore, MD, USA) with the sessions optimized via review and alteration by research staff using internet connection to the cycle (Figure 1). This process allowed research staff to review cycling duration, speed and resistance levels as well as the electrical stimulation parameters required during sessions to perform the exercise. This information allowed research staff to remotely alter electrical parameters to safely increase exercise duration, while maintaining preferred cycling speed and tolerated cycling resistance.

Before starting the program, the participant underwent a medical examination by a physician and successfully completed a trial FES-LEC session in a medical center SCI exercise laboratory. Although the participant's diet and physical activity levels, other than the FES-LEC, were not monitored, the participant was asked to maintain her regular diet and physical activity levels as major fluctuations in either could influence the results of the study.

\section{FES cycling parameters}

Adhesive gel electrodes were placed on the quadriceps, hamstrings and gluteal muscles for the transmission of coordinated bouts of electricity that induced the cycling activity. The electrical current amplitude was maintained at $140 \mathrm{~mA}$, while pulse width ranged from $250-300 \mu$ s and frequency ranged from $33.3-50 \mathrm{~Hz}$. The resistance was tied to the cycling speed so that the set speed could continue throughout each cycling session. As the speed decreased in a given session due to muscle fatigue, the resistance would automatically decrease to allow the participant to maintain the set cycling speed. As the muscles conditioned, the participant was able to cycle longer at higher resistance levels. The initial average resistance tolerated per session was $0.64 \mathrm{Nm}$. This doubled to $1.28 \mathrm{Nm}$ over the course of the year-long study. The cycling speed maintained between 36 and $43 \mathrm{rpm}$. The initial speed and

${ }^{1}$ Hunter Holmes McGuire VA Medical Center, Spinal Cord Injury and Disorders Center, Richmond, VA, USA and ${ }^{2}$ Physical Medicine and Rehabilitation Department, Virginia Commonwealth University, Richmond, VA, USA

Correspondence: Dr DR Dolbow, University of Southern Mississippi, 4124W 4th St., \#2407, 118 College Drive, \#5142, Hattiesburg, MS 39401, USA.

E-mail: d.dolbow@aol.com

Received 28 October 2013; revised 3 March 2014; accepted 6 March 2014 


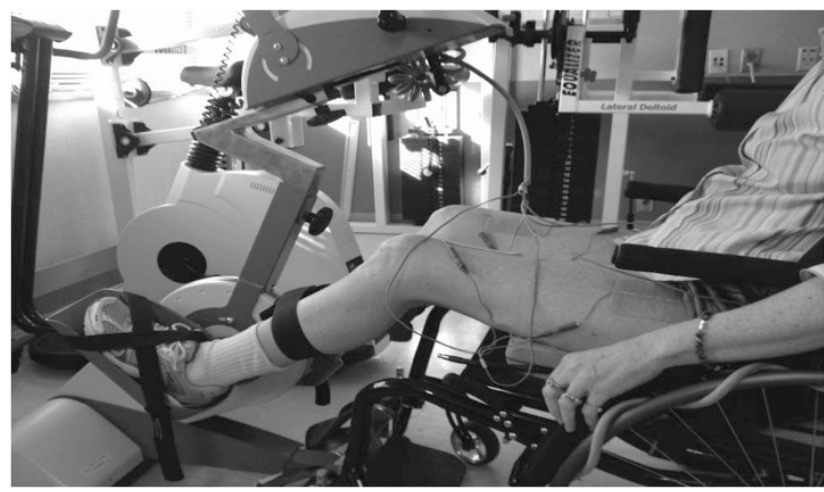

Figure 1 FES cycle with leads going to surface electrodes on participant's legs.

resistance were set according to participant comfort and the ability to maintain the cycling activity for $20 \mathrm{~min}$. The duration was gradually increased to a maximum of $63 \mathrm{~min}$ including a $2 \mathrm{~min}$ warm-up and $1 \mathrm{~min}$ cool-down per cycling session.

\section{Assessment}

Lean mass (LM), FM and bone mineral density (BMD) were measured before and after the 12 months of FES-LEC using the same Lunar Prodigy Advance Dual-Energy X-ray Absorptiometry (DXA) Scanner (General Electric, Madison, WI, USA) and the same trained DXA technician. The coefficient of variability of repeated scans at our facility has been determined to be less than $3 \%{ }^{4}$

\section{RESULTS}

Over the 12-month-study, there was a 7.7\% increase in total body LM and a $4.1 \%$ increase in legs LM. The total body weight was essentially unchanged although there was a $1.2 \%$ decrease in total body FM and a $9.9 \%$ decrease in legs FM. Whole-body BMD was increased from 0.934 to 1.023 (Table 1). Overall, there was a decrease in \% body fat from 48.4 to 46.3 and an improvement in the DXA T-score from -2.4 to -1.3 .

\section{DISCUSSION}

Although both of the pre and post percent body fat scores were in the unhealthy range, ${ }^{5}$ the increases in total and legs LM and the corresponding decreases in total and legs FM support the findings of Griffin et al. as well as two earlier case studies on males after
Table 1 Changes in body composition

\begin{tabular}{|c|c|c|c|c|c|c|c|c|c|c|}
\hline & $\begin{array}{l}W t \\
(\mathrm{~kg})\end{array}$ & $\begin{array}{c}H t \\
(\mathrm{~cm})\end{array}$ & $B M I$ & $\begin{array}{l}\% \\
B F\end{array}$ & $\begin{array}{l}L M \\
(\mathrm{~kg})\end{array}$ & $\begin{array}{l}\text { LLM } \\
(\mathrm{kg})\end{array}$ & $\begin{array}{l}F M \\
(\mathrm{~kg})\end{array}$ & $\begin{array}{l}\text { LFM } \\
(\mathrm{kg})\end{array}$ & $B M D$ & $\begin{array}{c}\mathrm{T}- \\
\text { score }\end{array}$ \\
\hline Pre & 80.42 & 154.94 & 33.5 & 48.3 & 39.22 & 12.34 & 36.79 & 14.91 & 0.934 & -2.4 \\
\hline Post & 80.92 & 154.94 & 33.7 & 46.3 & 42.23 & 12.85 & 36.35 & 13.43 & 1.023 & -1.3 \\
\hline
\end{tabular}

9 weeks and 6 months of FES-LEC reported by our laboratory. ${ }^{6,7}$

These results also enhance the theory that regular long-term FES-LEC may decrease the risk factors associated with cardiovascular and metabolic diseases. The fact that BMD increased supports the findings of Frotzler et al. ${ }^{3}$ and provides hope that this activity may also help enhance bone health.

The results of this case report cannot be generalized to the overall population of those with SCI; however, it does indicate that these beneficial results are possible. Further study with a large sample is needed to substantiate the findings of this case report. There also needs to be more extensive study concerning BMD in the areas of highest risk for decreased bone mass and fracture after SCI, that is, distal femur, proximal and distal tibia.

\section{CONFLICT OF INTEREST}

The authors declare no conflict of interest.

1 Gater DR, Dolbow D, Tsui B, Gorgey AS. Functional electrical stimulation therapies after spinal cord injury. NeuroRehabilitation 2011; 28: 231-248.

2 Griffin L, Decker MJ, Hwang JY, Wang B, Kitchen K, Ding Z et al. Functional electrical stimulation cycling improves body composition, metabolic and neural factors in persons with spinal cord injury. J Electromyogr Kinesiol 2009; 19: 614-622.

3 Frotzler A, Coupaud S, Perret C, Kakebeeke TH, Hunt KJ, Donaldson N et al High-volume FES-cycling partially reverses bone loss in people with chronic spinal cord injury. Bone 2008; 43: 169-176.

4 Gorgey AS, Chiodo AE, Zemper ED, Hornyak JE, Rodriguez GM, Gater DR. Relationship of spasticity to soft tissue body composition and the metabolic profile in persons with chronic motor complete spinal cord injury. J Spinal Cord Med 2010; 33: 6-15.

5 American College of Sports Medicine. ACSM's Guidelines for Exercise Testing and Prescription, 7th Edn, Lippincott Williams \& Wilkins Publishing Co, 2006; pp 68.

6 Dolbow DR, Gorgey AS, Cifu DX, Moore JR, Gater DR. Feasibility of home-based functional electrical stimulation cycling: case report. Spinal Cord 2012; 50: 170-171.

7 Dolbow DR, Gorgey AS, Moore JR, Gater DR. A report of practicability of a six month home based functional electrical stimulation cycling program for an individual with tetraplegia. J Spinal Cord Med 2012; 35: 182-186. 